\title{
Effects of Thermal Roughness on X-ray Studies of Liquid Surfaces
}

\section{Citation}

Pershan, Peter S. 2000. Effects of thermal roughness on x-ray studies of liquid surfaces.

Colloids and Surfaces A 171(1-3): 149-157.

\section{Published Version}

doi:10.1016/S0927-7757(99)00557-9

\section{Permanent link}

http://nrs.harvard.edu/urn-3:HUL.InstRepos:4455229

\section{Terms of Use}

This article was downloaded from Harvard University's DASH repository, and is made available under the terms and conditions applicable to Other Posted Material, as set forth at http:// nrs.harvard.edu/urn-3:HUL.InstRepos:dash.current.terms-of-use\#LAA

\section{Share Your Story}

The Harvard community has made this article openly available.

Please share how this access benefits you. Submit a story.

Accessibility 


\title{
Effects of thermal roughness on X-ray studies of liquid surfaces
}

\author{
P.S. Pershan* \\ Physics Department and the Division of Engineering and Applied Sciences, Harvard University, 205C Pierce Hall, 29 Oxford Str., \\ Cambridge, MA 02138, USA
}

\begin{abstract}
The effects of thermal roughness on X-ray studies of liquid surfaces will be discussed. In contrast to solid surfaces, for which the surface height-height correlation function remains finite at large distances, for liquid surfaces thermal fluctuations induce a logarithmic dependence for distances smaller than a gravitationally induced cutoff that is of the order of $\mathrm{mm}$. As a result of this there is no true specular X-ray reflectivity liquid surfaces. Theory and measurements explaining this phenomena will be presented. (C) 2000 Elsevier Science B.V. All rights reserved.
\end{abstract}

Keywords: X-ray; Liquid; Cutoff

\section{Introduction}

In recent years X-ray reflectivity (XR) has increasingly been demonstrated to be an important technique for characterization of surface structure. Although the application of X-ray reflectivity to solids is relatively straightforward, as a consequence of the two-dimensional character of thermal fluctuations in the height of the liquid surface, the application to liquids is somewhat subtler. In this article we will discuss some of the fundamental differences between X-ray reflectivity

* Tel.: + 1-617-4953214; fax: + 1-617-4959837.

E-mail address: pershan@deas.harvard.edu (P.S. Pershan) from solid and liquid surfaces. We will also explain that, as a result of thermally excited capillary fluctuations of the height of the liquid surface, there is a limit to the largest incident angles at which specular reflectivity from a liquid surface can be said to exist. Finally, we will demonstrate that the effect of thermal capillary waves can be understood quantitatively in terms of a relatively simple model and we will show that because the phenomena is so well understood it should be possible to separate the effects of capillary roughness from other sources of surface diffuse scattering.

The principal goal of measuring X-ray reflectivity, $R(\alpha)$, is to obtain a description of the average electron density profile, $\langle\rho(z)\rangle$, along the surface normal, which we take to be the $z$-axis. For X-ray reflectivity the 'master formula': 


$$
\begin{aligned}
R(\alpha) & \approx R_{\mathrm{F}}(\alpha)\left|\frac{1}{\rho_{e}(\infty)} \int_{-\infty}^{\infty} \mathrm{d} z \frac{\mathrm{d}\left\langle\rho_{e}(z)\right\rangle}{\mathrm{d} z} \exp \left[i Q_{z} z\right]\right|^{2} \\
& \equiv R_{\mathrm{F}}(\alpha)\left|\Phi\left(Q_{z}\right)\right|^{2}
\end{aligned}
$$

that relates $R(\alpha)$ to $\langle\rho(z)\rangle$ was originally proposed by Als-Nielsen and the author in order to model the electron density profile associated with smectic order that is induced by the free surface of a liquid nematic crystal [1,2]. In this expression $R_{\mathrm{F}}(\alpha)$ is the theoretical Fresnel reflectivity for $\mathrm{X}$-rays incident at an angle $\alpha$ to an ideal flat abrupt interface between vacuum (or low density vapor) and a bulk phase with the average electron density, $\rho_{\varepsilon}(\infty)$, of the bulk material. Although only an approximation this expression for $R(\alpha)$ is accurate so long as $\alpha \gg \alpha_{\mathrm{c}} \equiv\left[\rho_{e}(\infty) r_{e} \lambda^{2} / \pi\right]^{1 / 2}$ where $\alpha_{\mathrm{c}}$ is the critical angle for total external reflection. When the inequality does not hold $\alpha$ must be corrected to account for refraction effects on passing from the low density vapor into the material. The quantity $\Phi\left(Q_{z}\right)$ defined by Eq. (1) is the local structure factor of the surface. For Xrays of wavelength $\lambda$ and $\alpha \geq 5 \alpha_{c}$ the wavevector transfer along the surface normal is, as indicated in Fig. $1, Q_{z}=(4 \pi / \lambda) \sin (\alpha)$. In view of the fact that all information on the phase is lost on taking the absolute value of the integral in Eq. (1) it is not possible to formally invert $\Phi\left(Q_{z}\right)$ to extract a unique $\langle\rho(z)\rangle$ from a measured $R(\alpha)$ [3]. On the other hand if one has a reasonable idea of the principal physical features of the surface it is

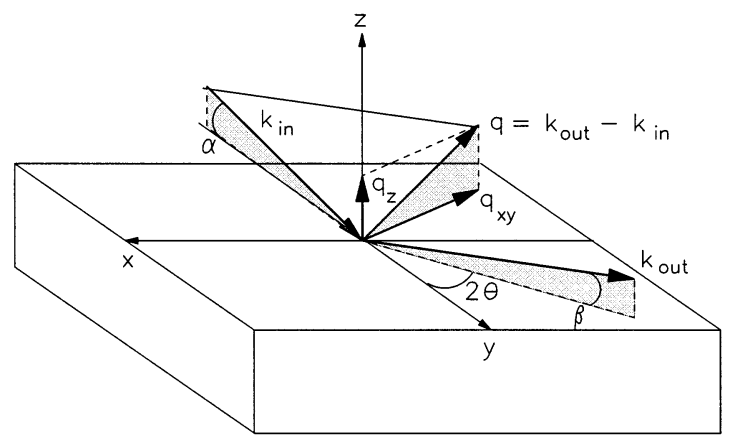

Fig. 1. Kinematics of X-ray scattering from liquid surfaces. Synchrotron radiation of wavelength $\lambda$ is incident at an angle $\alpha$ within the $y-z$ plane to the $x-y$ plane of the surface. The detector is positioned to probe angles $\beta$ to the surface and $\theta$ to the $y-z$ plane of incidence. possible to numerically fit realistic models of $\langle\rho(z)\rangle$ to the measured $R(\alpha)$.

\section{Scattering from solid surfaces}

The physical property that most makes X-ray reflectivity a simple tool for a flat solid surfaces is the fact that the characteristic length for correlations in the normal displacement of the surface height, $h\left(\mathbf{r}_{x y}\right)$, at points $\mathbf{r}_{x y} \equiv\{x, y\}$ and $\mathbf{r}_{x y}^{\prime}=$ $\left\{x^{\prime}, y^{\prime}\right\}$ is of sufficiently short range that for separations larger than some microscopic correlation length the mean square difference in height between two points $\mathbf{r}_{x y}$ and $\mathbf{r}_{x y}^{\prime}\left\langle\left[h\left(\mathbf{r}_{x y}\right)-h\left(\mathbf{r}_{x y}^{\prime}\right)\right]^{2}\right\rangle$, becomes independent of $\left|\mathbf{r}_{x y}-\mathbf{r}_{x y}^{\prime}\right|$. This is both the necessary and sufficient condition for validity of the elementary Fresnel reflection law (i.e. the incident and reflected angles are equal and in the same plane). This can be derived formally by considering an approximate expression for the differential cross section for scattering of an X-ray of wavelength $\lambda$ incident on a surface at angle $\alpha$

$$
\begin{aligned}
\frac{\mathrm{d} \sigma}{\mathrm{d} \Omega} \approx & \frac{A_{0}}{\sin (\alpha)}\left(\frac{e^{2} \rho_{e}(\infty)}{4 \pi m_{e} c^{2}}\right) \\
& \times \frac{\left|\Phi\left(Q_{z}\right)\right|^{2}}{Q_{z}^{2}} \int_{\left|\mathbf{r}_{x y}\right|>\xi} d^{2} \mathbf{r}_{x y}\left\langle e^{\left\{i Q_{z}\left[h\left(\mathbf{r}_{x y}\right)-h(0)\right]\right\}}\right\rangle \\
& \times e^{\left[i Q_{x y} \mathbf{r}_{x y}\right]}
\end{aligned}
$$

where $\left\{e, m_{e}\right\}$ are the electron \{charge, mass\}, $\rho_{e}(\infty)$ is the electron density of the bulk, and $A_{\mathrm{o}}$ is the cross sectional area of the incident beam. Assuming that the statistical distribution of $\left\{h\left(\mathbf{r}_{x y}\right)\right\}$ obeys Gaussian statistics such that $\left\langle\left[h\left(\mathbf{r}_{x y}\right)-h(0)\right]^{2}\right\rangle$ approaches a finite value $2\left\langle h(0)^{2}\right\rangle$ as $\left|\mathbf{r}_{x y}\right| \rightarrow \infty$ one can demonstrate that the effect of the height variations on the reflectivity is similar to the Debye-Waller effect that thermal phonons have on Bragg reflection from a 3D crystalline lattice:

$$
\begin{aligned}
& \frac{\mathrm{d} \sigma}{\mathrm{d} \Omega} \approx \frac{A_{0}}{\sin (\alpha)}\left[\frac{e^{2} \rho_{e}(\infty)}{4 \pi m_{e} c^{2}}\right]^{2} \\
& \frac{\left|\Phi\left(Q_{z}\right)\right|^{2}}{Q_{z}^{2}} e^{-Q_{z}^{2}\langle h(0)\rangle^{2}} 4 \pi^{2} \delta^{2}\left(\mathbf{Q}_{x y}\right)
\end{aligned}
$$


In this $\mathbf{Q}_{x y}=\left\{Q_{x}, Q_{y}\right\}$ is the projection of the wavevector transfer into the $x-y$ surface plane:

$Q_{x}=(2 \pi / \lambda) \cos (\beta) \sin (2 \theta)$

$Q_{y}=(2 \pi / \lambda)(\cos (\alpha)-\cos (\beta) \cos (2 \theta))$

and $\delta^{2}\left(\mathbf{Q}_{x y}\right)=\delta\left(Q_{x}\right) \delta\left(Q_{y}\right)$ is a two-dimensional delta-function. The condition that $\left\{Q_{x}, Q_{y}\right\}=0$ is equivalent to the requirement of the Fresnel law of reflection that $\alpha=\beta$ and $\theta=0$. The local surface structure factor, $\Phi\left(Q_{z}\right)$ is defined in terms of an electron density profile $\left\langle\rho_{e}(z)\right\rangle$, that is averaged over a microscopic area parallel to the plane of the surface. Averaging is necessary since roughness on an atomic length scale due to fluctuations in atomic positions can not be distinguished from the roughness due to the atomic size.

The essential role of $\delta^{2}\left(\mathbf{Q}_{x y}\right)$ in the cross section (Eq. (3)), is to provide a tool for distinguishing between scattering from the bulk liquid and the surface. To be able to do this it is necessary for the resolution length of the spectrometer to be large in comparison with the correlation lengths $\xi$, for bulk sources of scattering. For example, if the detector is at a distance $L$ from the sample with a defining aperture that has half widths $\Delta w_{x} \times \Delta w_{y}$, the angular resolution $\Delta \theta \times \Delta \beta \approx$ $\left(\Delta w_{x} / L\right) \times\left(\Delta w_{y} / L\right)$ corresponds to wavevector resolution $\Delta Q_{x} \times \Delta Q_{y} \approx\{(2 \pi / \lambda) \Delta \theta,(2 \pi / \lambda) \sin$ ( $\beta$ ) $\Delta \beta$. Assuming that $(2 \pi / \lambda) \Delta \theta<<1 / \xi$, if $\theta$ is scanned across the specular condition the measured intensity will consist of a sharp reflectivity peak with a resolution limited width, superposed on top of a slowly varying background intensity due to bulk diffuse scattering. As can be seen from Eq. (3), surface roughness will reduce the intensity of the specular reflectivity peak by $\exp \left[-Q_{z}^{2}\left\langle h(0)^{2}\right\rangle\right]$ but it will not effect the width of the peak. The specular signal is the difference between the intensity measured at the specular condition $(\theta=0)$ and the intensity measured at some small, but finite $\theta$ outside of the detector resolution.

As will be discussed in the following section, liquid surfaces differ from solids in that thermal excitation of surface capillary waves induce a logarithmic dependence to the height-height correlations, $\quad<\left[h\left(\mathbf{r}_{x y}\right)-h\left(\mathbf{r}_{x y}^{\prime}\right)\right]^{2}>\sim \ln \left(\left|\mathbf{r}_{x y}-\mathbf{r}_{x y}^{\prime}\right|\right) \quad$ [4].
The purpose of this paper is to discuss the implications of this complication for the study of reflectivity from liquid surfaces.

\section{Scattering from liquid surfaces}

The constraints on atomic positions associated with the crystalline structure of solids, and which serve to keep the facets on solid surface flat on an atomic scale, are absent for fluids. As a result the two-dimensional energy density associated with fluctuations in the height of a liquid surface has the form:

$U=\frac{1}{2} g \rho_{\mathrm{M}} h\left(\mathbf{r}_{x y}\right)^{2}+\frac{\gamma}{2}\left\{\left(\frac{\partial h\left(\mathbf{r}_{x y}\right)}{\partial y}\right)^{2}+\left(\frac{\partial h\left(\mathbf{r}_{x y}\right)}{\partial y}\right)^{2}\right\}$

where $\rho_{\mathrm{M}}$ is the mass density and $\gamma$ is the surface tension of the liquid. In the same way that the Debye wavevector is introduced in the phonon theory of solids to limit the number of modes in a crystal to a number of the order of the number of atoms, it is natural to limit the maximum wavevector for surface capillary waves to a value $Q_{\max }$ that is of the order of the reciprocal of the interatomic distance $[5,6]$. One can show that this form of the energy density implies that the thermal equilibrium height-height correlation function has the form:

$\left\langle\left[h\left(\mathbf{r}_{x y}\right)-h\left(\mathbf{r}_{x y}^{\prime}\right)\right]^{2}\right\rangle \approx \frac{k_{B} T}{\pi \gamma} \ln \left(\frac{1}{Q_{\max }\left|\mathbf{r}_{x y}-\mathbf{r}_{x y}^{\prime}\right|}\right)$

for separation distances $\sqrt{\gamma / g \rho_{M}}(\mathrm{~mm}) \gg \mid \mathbf{r}_{x y}-$ $\mathbf{r}_{x y}^{\prime} \mid \gg 1 / Q_{\max }(\AA)$. As a result of the logarithmic height correlations of liquid surfaces, the product of the Debye-Waller like term and the $\delta^{2}\left(\mathbf{Q}_{x y}\right)$ term in the differential cross section (Eq. (3)) for a solid surface is replaced by an algebraic form with a cusp like singularity centered at: $\mathbf{Q}_{x y}=$ $\left\{Q_{x}, Q_{y}\right\}=0$.

$\frac{\mathrm{d} \sigma}{\mathrm{d} \Omega} \approx \frac{A_{o}}{\sin (\alpha)}\left(\frac{e^{2} \rho_{e}(\infty)}{m_{e} c^{2}}\right)^{2}\left(\frac{k_{B} T}{\gamma}\right) \frac{1}{Q_{x y}^{2}}\left(\frac{Q_{x y}}{Q_{\max }}\right)^{\eta}$

$\left|\Phi\left(Q_{z}\right)\right|^{2}$

where 

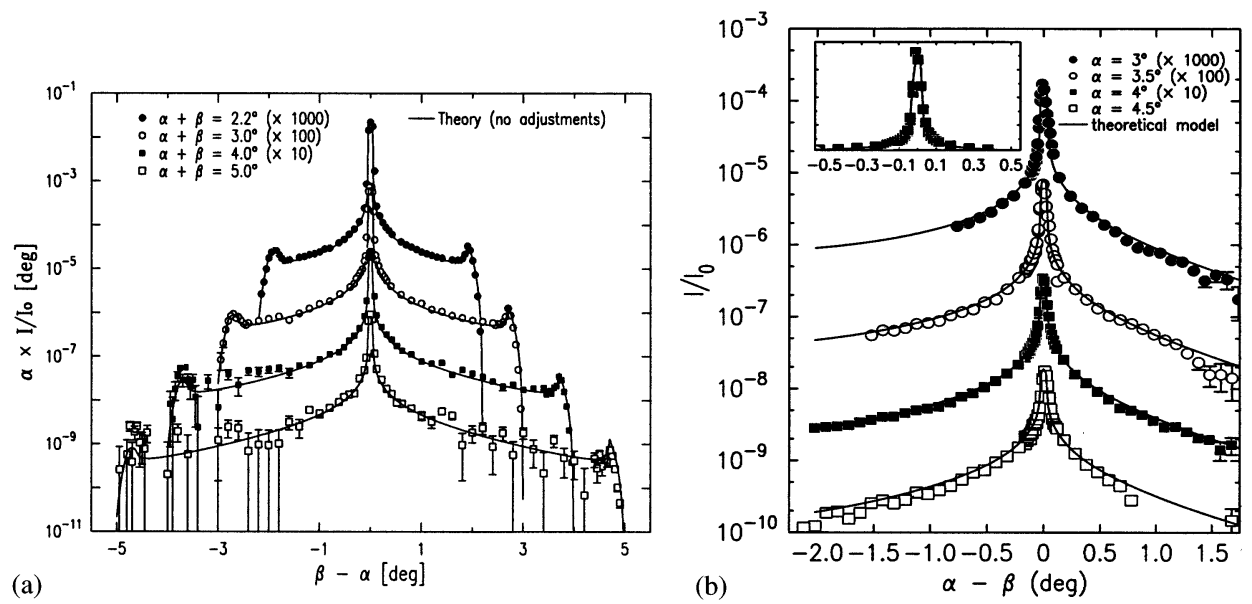

Fig. 2. Diffuse scattering from (a) the surface of water at room temperature and (b) liquid In at $170^{\circ} \mathrm{C}$.

$\eta=\frac{k_{\mathrm{B}} T}{2 \pi \gamma} Q_{z}^{2}$

This algebraic shape for the cross section implies that the simple procedure that was used for differentiating between the surface and bulk scattering of solids can not be applied to liquid surfaces. Below we will discuss a practical method that can be used for liquid surfaces; however, for the moment we will proceed as though the background scattering is negligible. In fact this is usually the case for small incident angles where bulk diffuse scattering is negligibly small in comparison with surface scattering.

In the mean time note that it is only if the quantity $\eta=k_{\mathrm{B}} T Q_{z} 2 /(2 \pi \gamma)<2$ that $\mathrm{d} \sigma / \mathrm{d} \Omega$ displays the cusp algebraic singularity at $Q_{x y} \approx 0$ and makes is possible to distinguish surface scattering from scattering originating in the bulk of the liquid. Physically this is because for sufficiently small $Q_{z},\left\langle\exp \left\{i<h\left(\mathbf{r}_{x y}\right)-h(0) Q_{z}\right\}\right\rangle \propto r_{x y}^{\eta}$ (see Eq. (2)) varies slowly enough that the Fourier transform in Eq. (2) is peaked at $Q_{x y} \approx 0$. For larger $Q_{z}$, or for $\eta \geq 2$, the primary contributions to the integral are weighted towards small $r_{x y}$ and $\mathrm{d} \sigma / \mathrm{d} \Omega$ is dominated by short distance, or atomic scale, correlations. The result for $\eta \geq 2$ is that both bulk and surface diffuse scattering are dominated by fluctuations on the same atomic length scale and there is no physical way to separate the two. Specular reflectivity from a liquid surface is only observable for $\eta \leq 2$. For practical experiments the limitation can even be more severe. For example, if the detector resolution $\Delta Q_{x}$ transverse to the plane of incidence is much broader than $\Delta Q_{y}$ within the plane of incidence, the integral of $1 / Q_{x y}^{2-\eta}$ over the $x$-component of the resolution function will result in a measured intensity as a function of $\beta$ (or $Q_{y}$ ) whose algebraic form could vary as slowly as $1 / Q_{y}^{1-\eta}$ In this case the reflectivity peak is confined to values of $Q_{z}$ for which $\eta \leq 1$. To the best of our knowledge this important limitation has not been discussed elsewhere in the literature.

The line shape and the effects of $\eta$ are illustrated in Fig. 2 for diffuse scattering measured from the surface of a dielectric liquid (water) and a liquid metal (In). In order to account for the non-surface diffuse or bulk diffuse scattering the measurements, and also the theory (solid lines), correspond to:

$$
\begin{aligned}
\Delta I(\alpha, \beta) \equiv & I(\alpha, \beta, 0) \\
& -(1 / 2)[I(\alpha, \beta, \Delta \theta)+I(\alpha, \beta,-\Delta \theta)]
\end{aligned}
$$

where $I(\alpha, \beta, \theta)$ is the sum of all intensity that falls within the acceptance solid angle of the detector when it is located at $(\alpha, \beta, \theta)$. The angular offset $\Delta \theta \approx 0.3$ to $0.4^{\circ}$, corresponding to $\Delta Q_{x}$ varying from the order of $0.03-0.06 \AA^{-1}$ depending on $\lambda$. The data for water (left panel) was taken by simultaneously varying $\alpha$ and $\beta$ in order to 
keep $\alpha+\beta$ (or $Q_{z}$ ) constant. For the largest value of $\alpha+\beta=5.0^{\circ}$ the value of $\eta \approx 0.17$ is much less than 2 and the deviations from the simple $Q_{x y}^{2}$ power law are not significant. The cusps are relatively sharp and the integral over the cusp at the specular position has approximately the same half width as the detector. The solid lines are calculated by numerically integrating Eq. (7) over the resolution function formed by a rectangular slit with no adjustable parameters [6]. The small peaks at the two extreme ranges of the scans are the 'Yoneida peaks' that occur when either the incident or scattered angles are equal to the critical angle for total external reflection [7].

The data in the right panel is from the surface of a liquid metal (In) at $170^{\circ} \mathrm{C}$. For this data $\alpha$ was held constant and $\beta$ was varied. The largest $Q_{z}$ obtained, corresponding to $\alpha=4^{\circ}$ and $\beta=5.5^{\circ}$ corresponds to $\eta \approx 0.5$ and the effects of finite $\eta$ are apparent. The solid lines were calculated by integrating Eq. (7) over the known resolution function. In this case the calculation requires knowledge of the local structure factor $\Phi\left(Q_{z}\right)$ which was determined from the measurements of the $Q_{z}$ dependence of the specular reflectivity, which is $I(\alpha, \alpha+\Delta \theta)+I(\alpha, \alpha-\Delta \theta)$ for $\Delta \theta \approx 0.3^{\circ}$ as a function of $Q_{z}$. We take this difference to be a measure of the $Q_{z}$ dependence of the specular reflectivity.

\section{Specular reflectivity from a liquid surface}

In view of the tails of the cusp like shape of the scattering cross section (i.e. when $\eta \leq 2$ ) from the liquid surface the separation between surface and bulk diffuse scattering can be somewhat arbitrary. We argue that so long as the bulk diffuse scattering varies slowly over an angular range comparable to the spectrometer resolution specular reflectivity can be interpreted as the difference between the intensity that is observed when the detector is centered on the nominal specular position and that observed when the detector is moved off of the specular condition by an amount larger than the spectrometer resolution. To simplify the discussion, for the moment we will neglect bulk diffuse scattering. So long as $\eta$ is $\ll 2$ the reflectivity $R\left(Q_{z}\right)$ can in principle be calculated by integrating the peak in the differential cross section at $Q_{x y}$ over the solid angle $\mathrm{d} \Omega$ subtended by the detector:

$R\left(Q_{z}\right)=\int_{\Delta \beta \Delta \theta} \frac{\mathrm{d} \sigma}{\mathrm{d} \Omega}=\frac{\lambda^{2}}{4 \pi^{2}} \frac{1}{\sin (\alpha)} \int_{\Delta^{2} Q_{x y}} \mathrm{~d}^{2} Q_{x y} \frac{\mathrm{d} \sigma}{\mathrm{d} \Omega}$

where $\Delta^{2} Q_{x y} \equiv\left(\Delta Q_{x}\right)\left(\Delta Q_{y}\right) \approx(2 \pi / \lambda)^{2} \sin (\alpha) \cos$ $(\alpha) \Delta \beta \Delta \theta$ the projection of the detector resolution onto the plane of the surface.

The detector solid angle most commonly used in experiments is defined by a rectangular shape discussed earlier; however, if we make the heuristic approximation of taking $\Delta^{2} Q_{x y}$ to be circular with a radius $Q_{\text {res }}$ Eq. (9) is easily integrated to obtain:

$R\left(Q_{z}\right) \approx \frac{16 \pi^{2} \rho_{e}(\infty)^{2} e^{4}}{m^{2} c^{4}}\left(\frac{1}{Q_{z}^{4}}\right)\left(\frac{Q_{\mathrm{res}}}{Q_{\mathrm{max}}}\right)^{\eta}\left|\Phi\left(Q_{z}\right)\right|^{2}$

There are two important features to note about this result.

First, if $Q_{\text {res }} \approx Q_{\max }$ the predicted reflectivity is identical to the Fresnel reflectivity expected from an ideal flat surface in which the electron density jumps abruptly from the vacuum to $\mathrm{p}(\infty)$ for the bulk liquid [2]:

$R\left(Q_{z}\right) \approx R_{\mathrm{F}}\left(Q_{z}\right) \frac{16 \pi^{2} \rho_{e}(\infty)^{2} e^{4}}{m^{2} c^{4}}$

where $R_{\mathrm{F}}\left(Q_{z}\right) \propto\left(1 / Q_{z}^{4}\right)$. The physical reason for this is as follows. The Fourier components of capillary roughness $h\left(Q_{x y}\right)$ with wavevector $Q_{x y}$ scatter X-rays away from the specular condition; however, if the acceptance solid angle of the detector is large enough to collect this radiation the measured reflectivity is not reduced by this scattering [8-10]. It follows that if the acceptance solid angle of the detector, as defined by $Q_{\text {res }}$, is large enough to collect scattering from all of the surface capillary waves the measured intensity should be the same as that of the ideal flat surface. Daillant et al. had discussed an alternative proposal to that of introducing a high $Q_{x y}$ cutoff to the integral from which Eq. (6) is derived. They prefer to assume that for short distances the energy density given by Eq. (5) is dominated by the 
higher order $\left[\partial^{2} h\left(\mathbf{r}_{x y}\right) / \partial x^{2}\right]^{2}$ [11]. This has the effect of changing the denominator in the integral to something of the form $\rho_{\mathbf{M}}+\gamma\left|\mathbf{Q}_{x y}\right|^{2}+K\left|\mathbf{Q}_{x y}\right|^{4}$ and serves to effectively cut off the integral at $Q_{x y}^{2} \approx \sqrt{\gamma / K}$ although this is just as sensible an approximation as the imposition of the hard cutoff at $Q_{\max }$, introduced here, unless there is short wavevector data to justify the $K\left|\mathbf{Q}_{x y}\right|^{4}$ term there is also no reason to prefer it. Furthermore we find it hard to justify assuming that terms of order $Q_{x y}^{4}$ are sufficiently large in comparison terms of order $Q_{x y}^{2}$ to cutoff the integral, while terms of order $Q_{x y}^{6}$ and higher order are negligible. The argument is similar to the wavevector expansions that form the basis for the rigorous underpinning of hyrdroynamics [12]. Furthermore, it seems that it would be very difficult to experimentally differentiate the $Q_{x y}^{4}$ algebraic form of the cutoff from others that might be suggested.

Second, in view of the fact that the projection of the height of the detector aperture onto the $x-y$ plane is proportional to $\sin (\alpha)$ the reflectometer resolution within the plane of incidence, $\Delta Q_{y} \approx(2 \pi / \lambda) \sin (\alpha) \Delta \beta \quad$ becomes increasingly

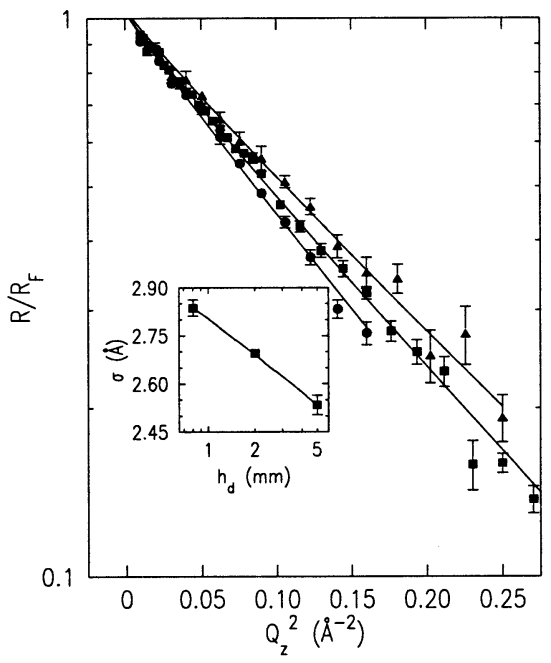

Fig. 3. Specular reflectivity data for water in the form $R\left(Q_{z}\right)$ / $R_{\mathrm{F}}\left(Q_{z}\right)$ vs. $Q_{z}^{2}$ for detector heights of $0.8 \mathrm{~mm}(\boldsymbol{\bullet}), 2.0 \mathrm{~mm}(\boldsymbol{\square})$ and $5.0 \mathrm{~mm}(\boldsymbol{\Delta})$. The solid lines represent the best fit to Eqs. (12a) and (12b). The inset shows the dependence of the average value of $\sigma$ on the vertical detector opening, kid. The slope of the connecting line is in perfect agreement with the predictions of Eq. (12b) if one assumes that $Q_{\text {res }} \propto \sin (\alpha)$. coarse as $Q_{z}=(4 \pi / \lambda) \sin (\alpha)$ increases. As a result, with increasing $Q_{z}$ more and more of the radiation that is scattered away from the specular direction falls within the acceptance solid angle of the detector. This is evident in the fact that $Q_{\text {res }} /$ $Q_{\max }$, which is less than unity, increases with increasing $Q_{z}$ and has the effect that for fixed detector slits the dependence of the reflectivity on $Q_{z}$ is slightly less rapid than the explicit dependence shown in Eq. (10) $[9,10]$.

Historically, most of the published treatments of these effects have been described by introducing a phenomenological resolution dependent surface roughness $\sigma^{2}(\alpha)$ such that [8]:

$R\left(Q_{z}\right) \approx R_{\mathrm{F}}\left(Q_{z}\right) \exp \left[-\sigma^{2}(\alpha) Q_{z}^{2}\right]$

where

$\sigma^{2}(\alpha) \approx\left(\frac{k_{B} T}{2 \pi \gamma}\right) \ln \left(\frac{Q_{\mathrm{max}}}{Q_{\mathrm{res}}}\right)=\frac{\eta}{Q_{z}^{2}} \ln \left(\frac{Q_{\mathrm{max}}}{Q_{\mathrm{res}}}\right)$

and $Q_{\text {res }}$ is proportional to $Q_{z}=(4 \pi / \lambda) \sin (\alpha)$. As a practical matter the logarithmic dependence of $\sigma^{2}(\alpha)$ on $\alpha$ can often be neglected. For example the dependence on $\alpha$ is clearly not important when $Q_{z}$ is small and $\exp \left(-\sigma^{2}(\alpha) Q_{z}^{2}\right) \approx 1$. The dependence on $\alpha$ only becomes important when $\sigma^{2}(\alpha) Q_{z}^{2} \approx 1$; however, the range of $\alpha$ between the value at which $\sigma^{2}(\alpha) Q_{z}^{2} \approx 1$ and the values when $\exp \left[-\sigma^{2}(\alpha) Q_{z}^{2}\right]$ becomes small enough that measurements become impossible is rarely larger than a factor of 3 or 4 . Over this range of $\alpha$, or $Q_{z}$, the logarithmic dependence can often be neglected. This can be seen in the data shown in Fig. 3 from Schwartz et al. [8]. The data shown is a $\log -\log$ plot of $\left[R\left(Q_{z}\right) / R_{\mathrm{F}}\left(Q_{z}\right)\right]$ vs. $Q_{z}^{2}$ for three different vertical openings of the aperture before the detector. As can be seen for each aperture the data is nearly linear, implying that the $\alpha^{2}$ dependence on $\alpha$ is nearly constant over the measured range of $0<Q_{z} \lesssim 0.5$. On the other hand, evidence of the effect of the size of the detector is illustrated in the inset by the expected linear dependence between the best fit values of $\sigma(\alpha)$ and the logarithm of the vertical aperture opening.

In a separate experiment Ocko et al. studied the temperature dependence of the specular reflectivity from a series of $n$-alkanes (C20 and C36). They made use of the fact that the surface tension 

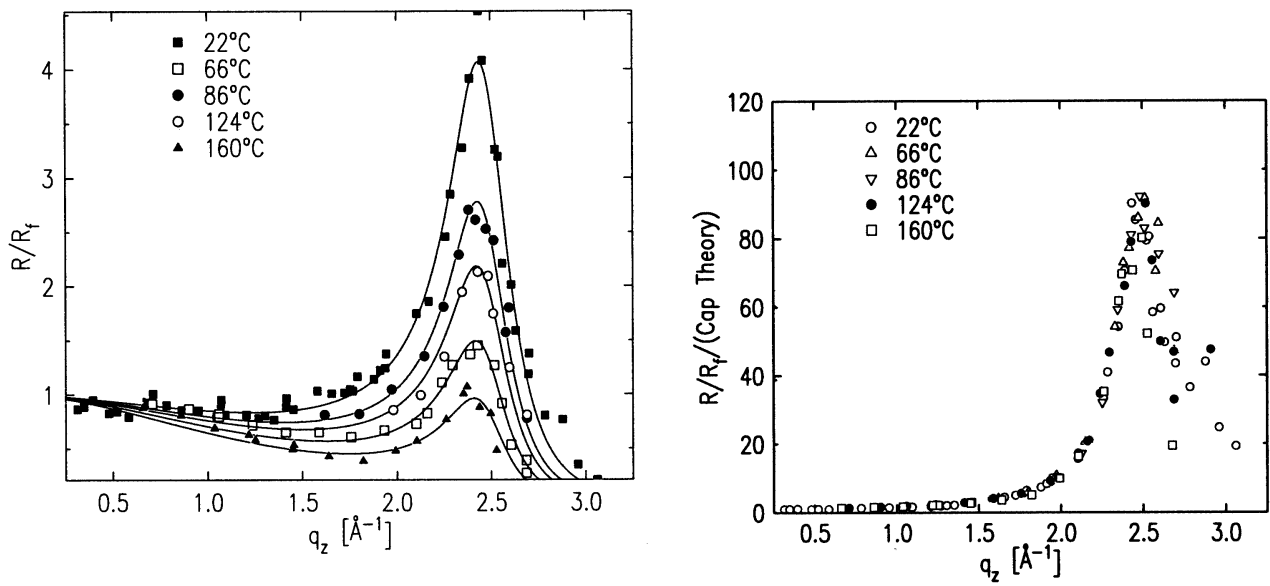

Fig. 4. (Left) normalized specular reflectivity data in the form of $R\left(Q_{z}\right) / R_{\mathrm{F}}\left(Q_{z}\right)$ from the surface of liquid Ga as a function of temperature. (Right) the same data as in the left panel divided by the predicted effect of thermal capillary waves $\left(1 / Q_{z}^{2}\right)\left(Q_{\text {res }} / Q_{\text {max }}\right)^{\eta}$.

of these alkanes is a strong function of temperature to demonstrate the validity of Eqs. (12a) and (12b) [13].

For liquid metals the combination of larger surface tension and larger electron density allows $\mathrm{X}$-ray reflectivity to be measured to values of $Q_{z} \approx 3 \AA^{-1}$ and the effect of capillary waves is much more dramatic. The left panel in Fig. 4 shows data for the ratio $\left[R\left(Q_{z}\right) / R_{\mathrm{F}}\left(Q_{z}\right)\right]$ from the surface of liquid $\mathrm{Ga}$ for a range of temperatures. As discussed elsewhere the atoms at the surface of liquid metals are expected to be arranged in layers parallel to the surface and this is confirmed by the quasi Bragg peak observed for $\mathrm{Ga}$ at $Q_{z} \approx$ $2.4 \AA^{-1}[14,15]$. The fact that all of this temperature dependence is due to thermal capillary waves is demonstrated in the right panel of Fig. 4 where we show the results of dividing the data on the left by $\left(Q_{\text {res }} / Q_{\max }\right)^{\eta}$. As can be seen the predicted effect due to thermal capillary waves completely accounts for all of the temperature dependence. Note that for liquid $\mathrm{Ga}$ at $160^{\circ} \mathrm{C}$ the value of $\eta \approx 1$.

\section{Diffuse scattering from inhomogeneous surfaces}

Finally we would like to point out that the diffuse scattering from thermal capillary waves on liquid surfaces is sufficiently well understood that
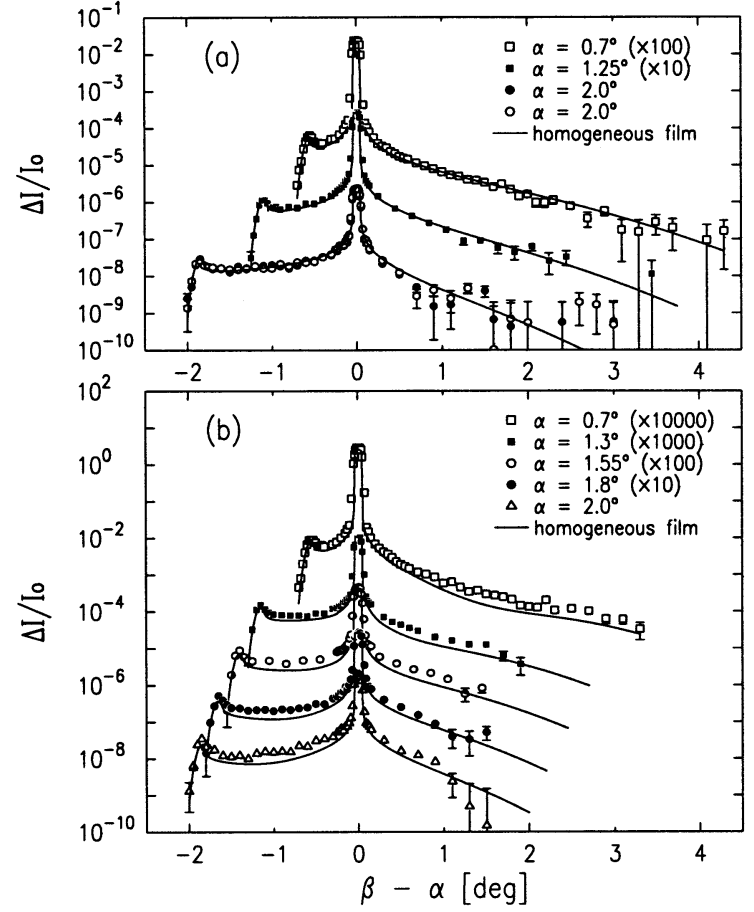

Fig. 5. Measured $\Delta I / I_{0}$ vs. $(\beta-\alpha)$ for PBLG (a) homogeneous Langmuir monolayer and (b) an inhomogeneous bilayer. The solid curves (-) are theoretically expected predictions for homogeneous PBLG films based on Eq. (7) [6]. 
any deviation between the experimental data and the capillary wave model can be interpreted in terms of surface inhomogeneities. The one published example of this approach is the experiment by Fukuto et al. on diffuse scattering from Lang-muir monolayers of the synthetic polypeptide, poly- $\gamma$-benzyl-L-glutamate (PBLG) on the surface of water [6]. The data in the upper panel of Fig. 5 illustrates diffuse scattering from a homogeneous monolayer of PBLG, the data in the lower panel shows data from similar measurements at higher surface density in which some of the molecules have been expelled into a second layer. Under conditions at which the data in the lower panel were taken the film is inhomogeneous. In both panels the solid lines represent the theoretical predictions calculated from Eq. (7) using the functional form for $\Phi\left(Q_{z}\right)$ that best fits the specular reflectivity $R\left(Q_{z}\right)$. For the homogeneous monolayer (upper panel) the data and theory agree perfectly. For the inhomogeneous layer (lower panel) the theoretical prediction of capillary wave theory is consistently below the data indicating excess scattering. This data has been analyzed and the correlation length for the surface inhomogeneity arising from two-dimensional clusters of molecules in the second layer is of the order of $1000 \AA$.

\section{Summary}

In this paper we have discussed some of the basic phenomena that must be considered in interpreting $\mathrm{X}$-ray scattering from the liquid surface. It has widely been recognized that surface roughness due to thermally excited capillary waves are responsible for a Debye-Waller like factor that reduces the intensity of specular reflectivity for larger angles. What has not previously been discussed; however, is the fact that for incident angles larger than the value for which the exponent $\eta=2$ it is not possible to distinguish surface and bulk scattering. This effect can be exasperated by the shape of the resolution function and for some situations the practical limit for measuring specular reflectivity from liquid surfaces might correspond to $\eta \approx 1$. Nevertheless, the effects of thermal capillary waves are well understood and detailed studies of in-plane correlations from liquid surfaces are possible for liquids with $\eta \leq 2$.

\section{Acknowledgements}

The research on capillary waves that formed the basis for this article was carried out in long standing collaboration with Moshe Deutsch(BarIlan) and Ben Ocko (BNL) and a series of younger collaborators that include Alan Braslau and Dan Schwartz. Two of these, Elaine DiMasi and Holger Tostmann have contributed one separate article to this issue and Mark Schlossman and his colleagues have submitted another. The research support was provided by grants to Harvard University by the National Science Foundation, NSF-DMR95-23440 and the Department of Energy DE-FG02-88-ER45379. Measurements were performed at the Harvard/ BNL Liquid Surface Spectrometer on Beam Line X22B and at X25 at the NSLS. Support for BNL is through DOE Contract No. DEAC02-98CH10886.

\section{References}

[1] J. Als-Nielsen, F. Christensen, P.S. Pershan, Phys. Rev. Lett. 48 (1982) 1107.

[2] P.S. Pershan, J. Als-Nielsen, Phys. Rev. Lett. 52 (1984) 759.

[3] P.S. Pershan, Phys. Rev. E50 (1994) 2369.

[4] S.K. Sinha, E.B. Sirota, S. Garoff, et al., Phys. Rev. B 38 (1988) 2297.

[5] H. Tostmann, E. DiMasi, P.S. Pershan, et al., Phys. Rev. B 59 (1999) 783.

[6] M. Fukuto, R.K. Heilmann, P.S. Pershan, et al., Phys. Rev. Lett. 81 (1998) 3455.

[7] Y. Yoneida, Phys. Rev. 131 (1963) 2010.

[8] D.K. Schwartz, M.L. Schlossman, E.H. Kawamoto, et al., Phys. Rev. A 41 (1990) 5687.

[9] A. Braslau, M. Deutsch, P.S. Pershan, et al., Phys. Rev. Lett. 54 (1985) 114.

[10] A. Braslau, P.S. Pershan, G. Swislow, et al., Phys. Rev. A 38 (1988) 2457.

[11] J. Daillant, L. Bosio, B. Harzallah, et al., J. Phys. II 1 (1991) 149. 
[12] P.C. Martin, O. Parodi, P.S. Pershan, Phys. Rev. A 6 (1972) 2401.

[13] H. Tostmann, E. DiMasi, B.M. Ocko, M. Dentsch, P.S. Pershan, J. Non-Cryst. Solids (1998) 250. (1999).
[14] H. Tostmann, E. DiMasi, B.M. Ocko, M. Dentsch, P.S. Pershan, J. Non-Cryst. Solids (1998) 250.

[15] S.A. Rice, Proc. Natl. Acad. Sci. USA 84 (1987) 4709. 